\title{
APPUNTI PER UNA EDIZIONE DEL GLOSSARIO BERGAMASCO MEDIOEVALE Di ANTONIO TIRABOSCHI
}

\author{
Federica Guerini ${ }^{1}$
}

\section{L'OPEROSO CANTIERE DI UN “MANOVALE DELLA CULTURA"}

Antonio Tiraboschi (1838 - 1883), una delle figure più attive e prolifiche sulla scena bergamasca nella seconda metà del XIX secolo, è noto soprattutto per la compilazione del Vocabolario dei Dialetti Bergamaschi Antichi e Moderni (1873), uno strumento di documentazione del patrimonio lessicale bergamasco non più eguagliato per mole, completezza e ricchezza delle fonti. La sua attività di ricerca, tuttavia, ha riguardato almeno tre ambiti disciplinari diversi, ancorché strettamente interrelati: la dialettologia, la storia e la demologia ${ }^{2}$.

L'interesse per la cultura e le tradizioni popolari è testimoniato sin dal 1864, quando viene dato alle stampe il saggio dal titolo Parre e il gergo dei suoi pastori, che coniuga competenze demologiche e linguistiche, in seguito approfondite nei saggi dedicati agli Usi pasquali nel bergamasco e agli Usi di Natale nel bergamasco, entrambi pubblicati nel 1878. Il prezioso corpus di Canti popolari bergamaschi, trascritti dal Tiraboschi nell'arco di una vita, è pubblicato postumo nel 1974, a cura di Roberto Leydi (cfr. Leydi, 1974). Sempre nel secolo scorso, grazie alla cura di Vittorio Volpi (cfr. Volpi, 1977), vedono la luce 37 fiabe inedite, 22 delle quali interamente in bergamasco, raccolte dallo studioso a varie riprese, presumibilmente negli anni appena precedenti la sua scomparsa, come sembra suggerire il contenuto di una lettera datata 26 aprile 1882 e inviata dallo studioso all'amico siciliano Giuseppe Pitré, nella quale si legge:

Le prometto di mandarle durante il corrente anno un saggio che rappresenti questa mia Bergamasca negli usi, nelle fiabe, nei proverbi, nelle canzoni, negli indovinelli, nei giochi fanciulleschi, insomma in tutto quello che ha di caratteristico questo mio nido, all'amore del quale ho sacrificato la mia carriera, ottenendo solo la soddisfazione di avere salvato molto di questo patrimonio avito.

\footnotetext{
${ }^{1}$ Università degli studi di Bergamo.

Il presente contributo si inserisce nell'ambito del Progetto PRIN 2017dal titolo Writing expertise as a dynamic sociolinguistic force: the emergence and development of Italian communities of discourse in Late Antiquity and the Middle Ages and their impact on languages and societies (Prot. 2017WLBK3Z), coordinato a livello nazionale dalla prof.ssa Piera Molinelli, che ringrazio della fiducia accordatami. Un sentito ringraziamento a Piera Molinelli e Francesco Lo Conte per avere letto e commentato una prima versione di questo lavoro, e ai due revisori anonimi, per il tempo dedicatomi e i preziosi suggerimenti, anche di carattere bibliografico. Chi scrive, naturalmente, deve considerarsi la sola responsabile delle eventuali inesattezze rimaste.

${ }^{2}$ La vita e le opere di Antonio Tiraboschi sono ampiamente descritte all'interno di lavori redatti sia da suoi contemporanei (ad es. Pitré, 1883; Prina, 1883; Mazzi, 1884; Mantovani, 1892), sia da studiosi vissuti nel secolo successivo (ad es. Poletti, 1942; Belotti, 1956; Volpi, 1977; Bondioli Magnati, Boninelli 1984; Boninelli, 1985; Passerini Tosi, 1985), ai quali si rimanda per una trattazione più organica e dettagliata dell'argomento. In questa sede, a beneficio del lettore, ci limiteremo a ricordare brevemente gli interessi di ricerca e alcuni dei lavori da lui pubblicati.
} 
A cavallo tra dialettologia e demologia, va ricordata l'importante raccolta di proverbi bergamaschi pubblicata nel 1875, all'interno della quale Tiraboschi sperimenta una particolare resa grafica del dialetto suggeritagli da Graziadio Isaia Ascoli, con il quale era da anni in contatto epistolare. L'insoddisfazione per le convenzioni di scrittura del dialetto, tuttavia, è già ravvisabile nell'edizione delle Poesie in dialetto bergamasco di Pietro Ruggeri da Stabello, uscita nel 1869 per i tipi della stamperia Pagnoncelli di Bergamo, nella quale Tiraboschi si discosta dalle convenzioni ortografiche adottate dal Ruggeri per introdurre un sistema di scrittura a suo avviso più coerente sul piano etimologico e, al tempo stesso, più trasparente per il lettore (Bondioli Magnati, 1984: 21).

L'esordio nell'ambito della dialettologia avviene nel 1859, a soli 21 anni, con un opuscolo intitolato Saggio di un vocabolario bergamasco (Tiraboschi, 1859), un lavoro di circa trenta pagine, limitato a un gruppo di lemmi inizianti con la lettera $A$, che dalle pagine della Gazzetta di Bergamo riceve il plauso di Gabriele Rosa e l'incoraggiamento a continuare la compilazione. Il Vocabolario comincia ad uscire in dispense presso la tipografia Bolis nel 1862 e, come già accennato, si completa nel decennio successivo.

Come molti vocabolari dialettali prodotti nel nostro paese durante la seconda metà dell'Ottocento, l'opera è motivata da un intento di carattere squisitamente didascalico ovvero l'insegnamento della «buona e viva odierna favella toscana» attraverso il dialetto, $\mathrm{o}$ più precisamente, $i$ dialetti bergamaschi. La scelta del plurale testimonia una consapevolezza della variazione diasistemica non limitata al titolo, ma ravvisabile in ogni pagina del Vocabolario, nel quale l'attenzione agli esiti più minuziosi della variazione diatopica accompagna il confronto tra il dialetto cittadino e le forme personalmente raccolte dal Tiraboschi nelle valli bergamasche (in val Gandino, valle Seriana, val di Scalve, valle san Martino, per citare solo alcuni esempi). Poco dopo la pubblicazione del Vocabolario, lo studioso viene invitato da Francesco Corazzini ad entrare a far parte della Società Dialettologica Italiana, un invito che accetta con riconoscenza, lieto di avere l'opportunità di «portare il [suo] sassolino ad un edificio che si prevede grandioso» (Bondioli Magnati, 1984: 24).

Il diploma in Paleografia conseguito nel 1874 presso la Scuola di Paleografia e Archivistica dell'Archivio di Stato di Milano (Bondioli Magnati, 1984: 24), permette al Tiraboschi di collaborare con Giovanni Finazzi all'edizione del più antico Statuto di Bergamo, il duecentesco Statutum vetus (cfr. \$2.2), e di riordinare le pergamene conservate presso la Biblioteca Civica Angelo Mai, dove ricopre il ruolo di bibliotecario a partire dal 1877. Le competenze di paleografo e archivista gli consentono inoltre di raccogliere come si legge in una lettera del 28 luglio 1876 indirizzata all'Ascoli - «scritture in dialetto bergamasco che, senza interruzione, forniscono saggi dal XIII secolo fino al presente». Queste ultime fanno parte del ricco e prezioso materiale inedito di cui il Glossario Bergamasco Medioevale si compone e che ancora attende di essere valorizzato.

\section{Il Glossario Bergamasco Medioevale}

\subsection{Struttura dell'opera}

Il Glossario Bergamasco Medioevale (d'ora in poi, Glossario), attualmente conservato presso la Biblioteca Civica Angelo Mai di Bergamo, è costituito da quattro quaderni manoscritti e inediti, all'interno dei quali sono registrati oltre 2000 lemmi organizzati alfabeticamente, secondo la seguente ripartizione:

- Quaderno I (segnatura MMB 23): lemmi da Abaregare a Bu₹us; 426 pagine, numerate a matita, nell'angolo esterno in alto, da mano diversa e verosimilmente posteriore, sino alla pagina 200 . 
- Quaderno II (segnatura MMB 24): lemmi da Ca 'casa' a Fustanus; circa 700 pagine, numerate a matita, nell'angolo esterno in alto, da mano diversa e verosimilmente posteriore, sino alla pagina 300 .

- Quaderno III (segnatura MMB 25): lemmi da Gabia a Runcum; 779 pagine, tutte numerate a matita, nell'angolo esterno in alto, da mano diversa e verosimilmente posteriore.

- Quaderno IV (segnatura MMB 26): lemmi da Sablo -nis a Zuvus; 590 pagine prive di numerazione.

In totale, i quattro quaderni comprendono all'incirca 2500 pagine, ma non tutte contengono degli appunti: Tiraboschi lascia intenzionalmente alcune pagine in bianco, come se prevedesse di includere altri lemmi in ordine alfabetico. Non è dato sapere quando ebbe inizio la raccolta di tali appunti, ma il confronto con i contenuti di alcuni lemmi del Vocabolario del 1873 (cfr. \3) lascia supporre che si tratti di un lavoro protrattosi per almeno un decennio. Molte annotazioni sono esplicitamente provvisorie: è evidente che lo studioso si proponeva di riprenderle in seguito, completandole e riformulandole ove necessario. La complessiva sistematizzazione del materiale raccolto sarà poi impedita dalle ristrettezze economiche e dal repentino peggioramento delle condizioni di salute del Tiraboschi, che avrà per esito la sua scomparsa, all'età di 45 anni, dopo solo quindici giorni di degenza presso una casa di cura alle porte di Bergamo.

Obiettivo di chi scrive è la creazione di una edizione digitale del Glossario, consultabile in modalità Open access a partire dalla home page della Biblioteca Civica Angelo Mai, e di una corrispondente edizione cartacea. Grazie ai contatti e alla collaborazione con questa Biblioteca, la digitalizzazione dei 4 volumi manoscritti di cui il Glossario si compone è già stata completata. Ciascuna immagine è stata ridenominata con il lemma (o i lemmi) in essa contenuti e numerata in modo progressivo; si è poi proceduto alla trascrizione dei contenuti di ogni singolo lemma al fine di creare una scheda da allegare a ciascuna immagine nell'ambito dell'edizione digitale. Attualmente si sta procedendo al controllo dei frammenti citati all'interno di ciascun lemma, attraverso il confronto sistematico con l'edizione della fonte, laddove essa sia disponibile, o in alternativa, nel caso di fonti inedite, mediante il riscontro dei manoscritti, al quale si prevede di affiancare un confronto con i contenuti di altre opere inedite dello stesso studioso - quali gli Appunti di grammatica comparata, di linguistica, di lessicologia (segnatura MMB 970), le raccolte di nomi locali medioevali (MMB 8-22) e di nomi personali medioevali (MMB 7), o la raccolta di vocaboli bergamaschi (MMB 738), tutte consultabili presso la Biblioteca Civica Angelo Mai.

\subsection{Fonti}

I frammenti citati da Tiraboschi allinterno dei diversi lemmi di cui il Glossario si compone provengono da un ampio ventaglio di fonti, edite ed inedite, che lo studioso indica accuratamente al temine di ogni citazione. Tra le più rilevanti possiamo citare gli Statuti di Bergamo, a cominciare dal duecentesco Statutum vetus (Biblioteca Civica Angelo Mai, ms. Sala I D 9, 21), edito da Giovanni Finazzi nel volume XVI della collana Historia Patric Monumenta (cfr. Finazzi, 1876), e pervenutoci mutilo in principio ed in fine, a cui fecero seguito gli Statuti del 1331 (Biblioteca Civica Angelo Mai, ms. Sala I D 9, 18), promulgati durante la breve dominazione di re Giovanni di Boemia e editi da Storti Storchi (1986). Anche dai cinque testi statutari redatti a Bergamo negli anni della

${ }^{3}$ Inizia infatti con la collatio VIII e termina con una parte della collatio XV, che doveva essere quella conclusiva (cfr. Padoa Schioppa, 1984: 45). 
dominazione viscontea - ovvero, seguendo Storti Storchi (1984: 51 e ss.), gli statuti del 1333 (Biblioteca Civica Angelo Mai, ms. Sala I D 9, 19) ${ }^{4}$, gli statuti del $1353^{5}$ (Biblioteca Civica Angelo Mai, ms. Sala I D 6, 1; editi da Forgiarini nel 1996), gli statuti del 1374 (Biblioteca Civica Angelo Mai, ms. Sala I D 7, 29), gli statuti del 1391 (Biblioteca Civica Angelo Mai, ms. Sala I D 9, 6) e quelli del 1422 (Biblioteca Civica Angelo Mai, ms. Sala I D 7, 34) - Tiraboschi attinge abbondantemente, dimostrando una profonda conoscenza delle aggiunte, delle stratificazioni e delle differenze intercorrenti tra i vari manoscritti ${ }^{6}$. La voce Graminosus, ad esempio, si apre con una breve descrizione del significato attribuibile a tale aggettivo («Infetto da malattia che i nostri veterinarj chiamano Gragnuola, i Toscani Panico, e che noi chiamiamo tuttora [in dialetto bergamasco, FG] grèmole; si manifesta in sulla cute con infiniti globetti di figura e di color panichino», corsivo nell'originale), seguita da una serie di attestazioni negli statuti cittadini che, dal duecentesco Statutum vetus, giungono sino al XV secolo:

(1) Sec. XIII: "Si contingerit beccarios $[. .$.$] emere porcos graminosos unum vel plures$ et verteretur in dubium si esset ille porcus qui venditus fuit graminosus an non" (St[atuti di] B[ergamo], XIII, 20).

An[no] 1331: "Et quod nulla persona debeat facere nec vendere aliquas carnes morbosas, gramilosas, sconflatas, vitiosas nec turpes" (St[atuti di] B[ergamo], VIII, 44).

Nello Stat[uto] del 1422 è scritto gramolosas ed in altro dello stesso secolo gramulosas

An[no] 1453: "Nulla persona vendere debeat aliquas carnes morbosas, gramlosas" (St[atuti di] B[ergamo], I, 184)7.

Una simile attenzione nel registrare le varianti grafiche che si alternano nei diversi testi statutari si osserva anche nei lemmi Glera ${ }^{8}$ (2) e $\operatorname{Vachetta}^{9}$ (3), per citare ancora solo un paio di esempi:

(2) An[no] 1249:

"Flumen Brembi sive glera vel ripa". (Mia racc[olta]).

$\operatorname{An}[\mathrm{no}]$ 1288:

"Ubi dicitur prope Geram".

An[no] 1353:

Et proiciatur terr "Et proiciatur terra super campis et lapides et gera proiciantur super strata" (St[atuti di] B[ergamo], XVI, 72).

Nel medesimo Statuto [collatio XVI], cap. 84, si legge: "Et proihicere terram super campis et gleram super stratis".

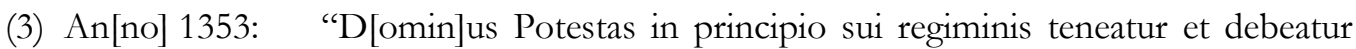
facere et habere et tenere unam vachettam de papiro seu unum librum qui dicitur vachetta". (St[atuti di] B[ergamo], IX, 2).

Negli Stat[uti] del 1391 e del 1422 si scrive vacheta.

\footnotetext{
${ }^{4}$ La storia della formazione di quest'ultimo Statuto - e, indirettamente, del precedente statuto del 1331 - è dettagliatamente descritta da Storti Storchi (2007), lavoro al quale qui si rimanda.

${ }^{5}$ Provvedimento legislativo noto anche come Statutum novissimum (Storti Storchi, 1984: 66).

${ }^{6}$ Più tardi, datati al 1453, sono gli Statuta datiorum, conservati ancora inediti in Bergamo, presso la Biblioteca civica Angelo Mai (ms. Sala I.D.7.28.9); ringrazio Francesco Lo Conte per questa informazione. Tiraboschi cita inoltre gli statuti del 1453 e quelli del 1491.

${ }^{7}$ Qui e in tutti gli esempi citati di seguito si mantiene il carattere corsivo come nell'originale. Le integrazioni apportate da chi scrive agli appunti manoscritti del Tiraboschi sono segnalate tra parentesi quadre.

8 'Ghiaia' (cfr. DELL \276), un lessema che, come mostra la seconda citazione al punto (2), dà origine a numerosi toponimi e micro-toponimi. Ringrazio uno dei revisori anonimi per questa preziosa segnalazione.

9 'Libro delle spese, dei conti' (cfr. REW \ 9109).
} 
Anche gli statuti rurali figurano tra le fonti alle quali Tiraboschi attinge. Si segnala in particolare lo statuto di Leffe, che Scharf (2004: 96) fa risalire all'anno 1272, tuttora conservato nell'Archivio comunale di Gandino ${ }^{10}$, un manoscritto che lo stesso Tiraboschi doveva conoscere dettagliatamente, avendone prodotto un'edizione a margine di un articolo nell'Archivio Storico Lombardo (cfr. Tiraboschi, 1882). Gli statuti di Vertova e quelli di Gandino sono pure citati in numerose occasioni.

Altri frammenti provengono poi dalle carte del Monastero di S. Sepolcro di Astino, la cui fondazione si presume avvenuta all'inizio del XII secolo. Delle carte risalenti agli anni 1101-1145 è disponibile un'edizione digitale a cura di Gianmarco Cossardi ${ }^{11}$, mentre le pergamene dei secoli successivi sono in larga misura ancora inedite.

Per quanto concerne i documenti riconducibili ad enti caritatevoli ed assistenziali, nel Glossario ricorrono citazioni tratte dalle pergamene conservate negli archivi delle Misericordie di Ardesio, di Gazzaniga e di Bergamo ${ }^{12}$. Vi sono inoltre un centinaio di frammenti al termine dei quali Tiraboschi appone l'indicazione "Mia raccolta", senza ulteriori precisazioni (cfr. es. 2). Anche se in questa fase non abbiamo ancora avuto modo di verificarlo in modo sistematico, possiamo ipotizzare che l'origine di tali frammenti debba ricercarsi nel Fondo Antonio Tiraboschi, conservato presso la Biblioteca Civica Angelo Mai, comprendente una serie di pergamene (nn. 3569-4008), databili dal X secolo in avanti. Si tratta di un cospicuo corpus di documenti relativi ai comuni di Vertova, Gazzaniga, Leffe e Gandino, tra cui numerosi atti giudiziari e ducali, nonché atti privati relativi a varie località della Val Brembana (parte dei quali provenienti dall'archivio del già citato Monastero di Astino), che Tiraboschi ebbe modo di raccogliere nel corso delle sue ricerche sul territorio bergamasco e che furono donate dagli eredi alla Biblioteca Civica in seguito alla sua morte ${ }^{13}$.

Vale la pena di notare che alcuni dei manoscritti citati da Tiraboschi all'interno del Glossario sono poi andati perduti. Un esempio emblematico è il cosiddetto Statutum vetus di Vertova - che Scharf (2004: 96) fa risalire all'anno 1235 - contenuto in un codice membranaceo che Tiraboschi rintracciò nell'autunno del 1863 e donò a Gabriele Rosa, il quale ne annunciò il ritrovamento pochi mesi dopo in appendice al suo Statuti inediti della Provincia di Bergamo (Rosa, 1863; cfr. Bondioli Magnati, Boninelli, 1984: 16; Cortesi, 1983 : 24-25). Possiamo conoscere tale statuto soltanto attraverso i frammenti citati nel Glossario e l'edizione ad opera del Rosa $(1869)^{14}$, poiché, in seguito alla morte di quest'ultimo, del prezioso codice si persero di nuovo, e sinora irrimediabilmente, le tracce.

\footnotetext{
10 Tale statuto, per la verità, non si è conservato integralmente: come osserva Scharf (2004: 96) «il codice originario era formato da tre quaderni rilegati insieme, di cui ne sono sopravvissuti solo due». In altre parole, è andato perduto circa un terzo del testo di cui lo statuto doveva essere composto.

${ }^{11}$ Cfr. Codice Diplomatico della Lombardia Medioevale, sec. VIII - XII:

http://www.lombardiabeniculturali.it/cdlm.

${ }^{12}$ Misericordie e confraternite laiche erano enti caritatevoli di assistenza documentati in Italia settentrionale dal principio del XII secolo; la tipologia di testi prevalente nelle pergamene di questo gruppo è quella degli atti notarili: vendite, permute, locazioni in territorio, passaggi di proprietà. Cfr. Little (1988) e Brolis, Brembilla, Corato (2001).

${ }^{13}$ Cfr. Zonca (2010: 17-18), il quale osserva che «il secondo volume di regesti della Biblioteca [Civica Angelo Mai, FG] (Ar 1/2), intitolato Catalogo delle pergamene possedute da A. Tiraboschi, pare compilato dallo stesso Antonio Tiraboschi per descrivere pezzi che erano ancora di sua proprietà, e successivamente donati alla Biblioteca insieme al detto Catalogo dalla famiglia dello studioso [...]. Il titolo stesso e la parte terminale della descrizione dei documenti si devono ad una mano diversa, forse quella di Angelo Mazzi, e sono collocabili appunto dopo l'avvenuta donazione alla Biblioteca».

14 Un'edizione che Scharf (2004: 96) descrive come «non priva tuttavia di notevoli errori di lettura». Lo stesso studioso aggiunge: «il testo del Rosa è stato ripubblicato a Vertova nel 1980 in edizione anastatica, rendendo conto in nota delle numerose correzioni e osservazioni apposte a una copia della prima edizione conservata nella Biblioteca di Bergamo e di mano del Tiraboschi, che aveva visto il codice originario» (ibidem).
} 
Da ultimo, ma certo non per importanza, meritano di essere menzionate le fonti "indirette", tra le quali spiccano per la quantità di frammenti citati le opere del canonico e storico bergamasco Mario Lupo, in primis i due volumi del Codex Diplomaticus Civitatis et Ecclesice Bergomatis, pubblicati rispettivamente nel 1784 e nel 1799, accanto al saggio Excerpta ex Actis Notariorum Bergomi, inedito, il cui manoscritto è conservato presso la Biblioteca Civica Angelo Mai di Bergamo ${ }^{15}$.

Ma come testimonia l'elenco delle opere citate dal Tiraboschi all'interno del Glossarioconsultabile nella prima sezione della Bibliografia a chiusura del presente contributo - lo studioso non si limita ad un collage di citazioni, ma arricchisce ciascun lemma con osservazioni e spunti di carattere linguistico, storico e demologico. Alla voce Buฉus 'alveare', ad esempio, leggiamo:

(4) Sec[olo] XV: "Item quod pro consuetudine servetur quod ex quolibet alveo seu brugazolo seu buгo apium". (St[atuti] di B[ergamo], III, 6) ${ }^{16}=$ Alveare.

Nelle nostre Valli di Scalve e Brembana ed in Piemonte si dice tuttora büs; nel Vicentino e nel Veronese dicono buzo de ave e nel Friuli boz des as; nella Leggenda di San Patrizio, codice in veneziano del sec. XV, si legge "le boze de la miel e de la cera piene de ave", e se crediamo a Romanin (Storia [Documentata] di Venezia, I, 228 e II, 52) i Veneziani chiamarono buzo un naviglio, laonde il famoso Buzindoro vorrebbe dire naviglio dorato. Da büs, boze buzo derivano i nostri diminutivi bozól, büzól e bizól = arnia, il bös che il nostro Assonica usò nel sign[ificato] di piffero, il béssola che nella V[alle] Calep[io] vale Zangola, il bozola dei nostri vecchi Statuti, il bossolo e bozzolo dell'ital[iano]. A me piace il trovare parentela tra il superbo Bucintoro e l'umile alveare.

Si notino il riferimento ad un codice quattrocentesco contenente la leggenda di San Patrizio, ai volumi I e II della Storia Documentata di Venezia (1853) di Samuele Romanin e al particolare impiego del bergamasco bös da parte di Carlo Assonica; i numerosi accenni alle varianti dialettali (piemontese, bergamasco, vicentino, veronese, veneziano e friulano) e il curioso commento apposto a chiusura del lemma ( $\mathrm{A}$ me piace il trovare parentela tra il superbo Bucintoro e l'umile alveare»).

Alla voce Sascire ${ }^{17}$, a commento di una citazione tratta da una delle pergamene donate alla Biblioteca Civica Angelo Mai dal sacerdote Luigi Femi, leggiamo invece: «Ł̀ notevole che in questa carta è stabilito che il creditore possa robare, sascire, etc. le robe del debitore fino a compiuto pagamento». E alla voce Albergaria: «era un obbligo molto pesante in forza del quale le popolazioni dovevano albergare almeno una volta all'anno il signore con tutti i suoi famigli o dargliene il compenso in denaro».

L'interesse del Tiraboschi per le tradizioni e la cultura popolare è evidente alla voce Corezola, nome di uno dei giochi vietati da numerosi statuti medioevali. Il lemma si apre con una serie di frammenti estrapolati dai testi statutari bergamaschi degli anni 1353-1491, ai quali fa seguito una citazione tratta dal secondo volume delle Dissertazioni sopra le antichità italiane di Ludovico Antonio Muratori, che chiarisce meglio la natura di tale gioco, con la precisazione:

Adunque la Corregiola e la Polverella dovean essere due differenti giuochi, che dai furbi erano proposti all'incauta plebe, per ismungere con facilità dagli sconsigliati, che osavano giuocare[,] il denaro. In Toscana Corregiu[o]lo altro

\footnotetext{
15 Segnatura AB 399.

16 Citazione tratta degli Statuti del 1453, f. 35v (ms. Sala I.D.9.8); un sentito ringraziamento a Francesco Lo Conte per la segnalazione.

17 'Occupare, impossessarsi' (cfr. REW S 7632).
} 
non è[,] che il Croginolo[,] o Crociuolo[;] e vi ha de' Ciurmadori che, con tre bussolotti fingendo di nascondere sotto l'un di essi un bottone, tirano alla trappola i goffi villani. Ma presso i Lombardi Corregiola è un dim[inutivo] di Correggia. Un prov[erbio] recato da Orlando Pescetti dice: Fare alla scorregginola; o ch'ella è dentro, o ch'ella è fuora.

Il lemma si chiude con la menzione delle varianti corizola, corezola, correzzola, corrizzola e corregiola, tutte attestate all'interno di testi statutari dell'Italia settentrionale.

Tra le tradizioni diffuse a livello locale, segnaliamo la voce Morgincap (cfr. Francovich Onesti 2013: 105; Jarnut 1980: 265), che si apre con la seguente annotazione:

Parola tedesca che significò dono del mattino ed era appunto secondo l'uso longobardo un dono fatto dal marito alla moglie nella prima mattina dopo le nozze. Quest'uso continuò fra noi fino in tempi non molto lontani ed anche a Firenze l'uso del morgincapo durò fino nei tempi più recenti. [...] Il $\mathrm{I}^{\circ}$ tomo del Codex Diplomaticus Cavensis si apre con una costituzione di morgengab dell'an[no] 792, di cui è dato il facsimile alla fine del tomo.

Estremamente interessanti, sia dal punto di vista lessicale, sia per gli studi di storia della cultura materiale nel medioevo, sono gli elenchi relativi a diversi tipi di abiti (gonella panni coloris da viro, gonella panni pergamaschi, gonella mulieris non scavezata, gonella scavezata cum uno bugetto et cum duobus bugettis, gonella ab homine), di giochi tradizionali (ludus bastonzellis, ludus paletti, ludus alee, bischazzie, reginete, ludus scachi, ludus stelle, ludus taxillorum, ecc...), di tessuti (pannus follatus, pannus sgrezzus, pannus samateyus aparigiatus, pannus francigenus gentilis, pannus florentinus de Tolosa, pannus bretinus aparegiatus, pannus blavetus, ecc...), di armi (cultelus ponctutus, frandolossus, spata, clipeus, tavolacius, boclerius, folzonus, cultellazollus, falx, misericordia, archus, balista, penatus, manarisius, securis, securisella, gratarolla de ferro, lancia ferri, rasgium ferri, sigzonus, inganolla, spitum ferri, mazza ferri, mazuchus feratus, bordonus, furcha ferri, forpix, stochus, cultellus a galono), per citare solo alcuni esempi.

Ma il contributo più prezioso che il Glossario offre al lettore risiede senza dubbio nelle osservazioni e nei commenti di natura linguistica, sociolinguistica e dialettologica rintracciabili in quasi ogni pagina, che saranno oggetto di una preliminare ricognizione all'interno del prossimo paragrafo.

\section{TIRABOSCHI DIALETTOLOGO E LESSICOGRAFO}

Gli interessi del Tiraboschi spaziavano, come si è accennato, dal folclore alla storia locale, dalla letteratura popolare ai gerghi, ma il contributo più incisivo si può senza dubbio ravvisare nell'ambito della dialettologia romanza e della lessicografia. E in effetti, nel Glossario, ricorrono numerose osservazioni e commenti di natura linguistica e dialettologica che, in questa fase ancora preliminare della ricerca, e tenuto conto del carattere provvisorio, da appunti di lavoro, delle annotazioni di cui il Glossario si compone, riteniamo possano essere distinti in tre sottogruppi:

i. note di carattere dialettologico, riguardanti gli esiti di forme latine o proto-romanze in italiano e nei dialetti gallo-italici (bergamasco, in primis) o relative alle particolari sfumature semantiche osservabili nel lessico del bergamasco;

ii. annotazioni di natura linguistica sulla comparsa di costruzioni romanze in sostituzione delle costruzioni latine, e sulle innovazioni semantiche romanze ravvisabili all'interno dei frammenti citati ad esemplificazione di ciascun lemma; 
iii. osservazioni di carattere onomastico, relative a toponimi, microtoponimi e - più raramente - nomi personali.

Le annotazioni riconducibili al primo sottogruppo sono senza dubbio le più frequenti e rivelano come molti appunti del Glossario siano confluiti nelle pagine del Vocabolario dei dialetti bergamaschi antichi e moderni, influenzando i lavori di lemmatizzazione e di compilazione che dovettero precederne la stampa. Il lemma Albius, ad esempio, si apre con la seguente osservazione: «Vasca, pila, gran vaso di pietra per ricevere l'acqua della fontana. L'odierno [bergamasco] albe è generalmente usato nel significato di trogolo, nel qual senso dicesi pure albio nel contado toscano, dal lat[ino] alveus»; nel Vocabolario (Tiraboschi, 1873: s.v. Albe) leggiamo: «Trogolo, vaso in cui si dà comunemente da mangiare ai polli [...]. L'alveus latino significava propriamente 'letto di fiume, alveo'; ma si usò pure per indicare un vaso da bagnarsi. Nel nostro Albe abbiamo conservato certamente qualche vestigio dell' alvens latino». La voce Conzare del Glossario si apre con una citazione tratta dagli statuti duecenteschi di Bergamo, seguita dalla nota: «i nostri mugnaj dicono tuttora cunsà nel sig[nificato] di crivellare il grano, ed anche gli Aretini dicono conciare»; nel Vocabolario, alla medesima entrata, leggiamo: «(Termine de' Mugnai) Crivellare il grano; gli Aretini dicono pure conciare [...]. In uno statuto di Bergamo del 1227: de frumento non mondo e non conciator. Si noti il raffronto, ravvisabile in molte pagine del Glossario, tra la forma bergamasca e l'esito toscano; a commento del lemma Abiaticus $^{18}$, Tiraboschi osserva come, in bergamasco, la coppia di lessemi biàdec 'nipote (di nonno)' e nè̀t 'nipote (di zio)' veicoli una distinzione semantica assente nella lingua nazionale:

In scritture del XV secolo ho trovato abiatico e abiadego e tuttora diciamo biàdec per nipotino, cioè nipote nato da figliolo o da figliola. è voce del Romancio e di tutta la Lombardia, ma non dell'italiano, che pur sarebbe necessaria per fuggire equivoci e circonlocuzioni.

Sec. XIII

"Et per dominum facta fuit vel fuerit investitura in filium vel habiaticum, fratrem vel nepotem". (St[atuti di] B[ergamo], X, 2).

An[no] 1331: "Ab ecclesia S[ancte] Marie usque ad domum q[uondam] Habiatici de Habiaticis". (St[atuti di] B[ergamo], IX, 6).

Qui è adoperato come nome proprio di persona e di famiglia.

A questo proposito, va osservato che il Glossario è pervaso da annotazioni di carattere semantico, che consentono di apprezzare la ricchezza del patrimonio lessicale bergamasco nella seconda metà dell'Ottocento, quando la vitalità dei dialetti non era ancora stata minacciata dalla diffusione della lingua nazionale. Alla voce Bretinus ${ }^{19}$, ad esempio, leggiamo: «Berrettino, di colore cenerognolo. Questo aggettivo si è conservato nel modo [di dire dialettale] vegn bretì, che adoperiamo nel sign[ificato] di adirarsi». L'entrata relativa all'aggettivo Cambratus ${ }^{20}$ si chiude invece con la seguente precisazione: «od[ierno] incambrät 'sprangato', da cambra, che significa pezzo di ferro con cui si tengono unite pietre con pietre». All'interno del lemma Manus leggiamo invece:

18 'Nipote (di nonno)' (REW \ 825).

19 'Di colore rosso' (REW \ 1117); tuttavia, come giustamente puntualizzato da uno dei revisori anonimi del presente contributo, l'etimologia del REW (< lat. birrus 'rosso, rossiccio'), risalente a Mussafia 1873, è stata rimessa in discussione da Cortelazzo a partire dal DELI (1979, s.v. berrettino) e sostituita con l'arabo bāruti, "del colore della polvere da sparo", "grigio".

20 'Sbarrato, sprangato' (REW \ 1564). 
(6) Sec. XIII: "Quam petiam terre idem D[omin]us Guilelmus tenebat ad suas manus". (Bibl[ioteca], perg[amena] N.N., 14).

Questo modo è spiegato dall'od[ierno] Fa 'nda la tèra da so ma, che vale 'far lavorare terre a sue spese, ritenendosi l'intiero raccolto'.

Il lemma Tremagium ${ }^{21}$ è glossato come «tramaglio, sorta di rete da uccellare che tuttodì chiamiamo tremăc, donde tremagì, il fabbricatore di reti». E gli esempi potrebbero continuare.

Tra le annotazioni riguardanti la comparsa di costruzioni romanze in sostituzione di costruzioni latine, si segnalano quelle relative allo sviluppo di forme analitiche (7), di preposizioni (8) e di verbi sintagmatici ${ }^{22}$, quali cazare fora 'scacciare' (9) o retro tornare 'restituire' vs. in retro tornare 'ritornare' (10), di cui Tiraboschi segnala con metodicità la prima attestazione nella documentazione di area bergamasca da lui consultata.

Avv[erbio] Là.

In la e I la. In là opposto di In qua.

An[no] 1172: "Et hoc fuit a triginta annis in za et a viginti in la". (Mia racc[olta]).

An[no] 1185: "A triginta annis in la (...) nisi a quatuor annis in za".

(Bibl[ioteca], rot[olo] 2312).

Da prepos[izione]

An[no] 795, An[no] 795, 805: "Est inter adfines da mane (...) da medio die et sera (...) 805: $\quad$ da montes". (Lupo, I, 638). È pure in carta bresciana dell'an[no] 795.

An[no] 886: "Que nobis advenerunt per scripta pagina da Arnaldo de vico Bussone". (Ib [idem], 983).

In nostro docum[ento] dell'an[no] 1152 (Bibl[ioteca], rot[olo] 2114) è ricordato un "Johannes dal monte", che ci fornisce esempio della stessa preposiz[ione] articolata.

(9) Cazare. Scacciare.

An[no] 1185: "Interrogatus si homines de Scano cazaverunt foras bestias illorum de Astino a triginta anni in la, vel a triginta annis infra, respondit Non, nisi a quatuor annis in $2 a^{\prime \prime}$.

(Bibl[ioteca], rot[olo] 2312).

(10) Retro tornare 'Restituire'.

An[no] 1244: "Et potestas teneatur condempnare eum in solidos decem imper[ialium] et retro tomare illos denarios illi a quo receperit". (St[atuti di] B[ergamo], XI, 15).

An[no] 1248: In retro tornare 'Ritornare'.

"Quelibet persona (...) possit ducere, menare (...) et in retro tornare sine aliquo tolloneo". (St[atuti di] B[ergamo], XIV, 13).

${ }^{21}$ Cfr. REW S 8875: *trèmaculum 'complesso di reti usate per la cattura di pesci o di altri animali selvatici'.

22 Seguendo la definizione adottata da Simone (1997: 156-57), chiamiamo verbi sintagmatici i «sintagmi formati da una testa verbale e da un complemento costituito da una 'particella' (originariamente un avverbio), uniti da una coesione sintattica di grado elelvato al punto che non si può commutare il verbo sintagmatico intero con una sola delle sue parti». 
(C) Italiano LinguaDue 2. 2021. F. Guerini, Appuntiper una edizione del Glossario Bergamasco Medioevale di Antonio Tiraboschi

Assai frequenti sono pure i riferimenti a inedite collocazioni semantiche romanze o innovazioni lessicali che Tiraboschi osserva nei documenti da lui consultati; apprendiamo così dell'esistenza di una distinzione tra ligna in pedibus, «legne in piedi, cioè non tagliate» $\mathrm{e}$ ligna menativa «legne che si possono menare, condurre da un luogo all'altro». Tra le unità di misura, è interessante citare l'asinata, corrispondente a 'tanta materia quanta ne può portare un asino', la caveda, che Tiraboschi ipotizza possa equivalere «al cavezzo bresciano (braccia 6), misura di terreni», il bigongio, nel quale riconosce il prefissoide bi-, seguito da congio, tradizionale misura per i liquidi, o ancora il somez२o, «odierno sómes, la lunghezza del pugno col dito grosso alzato».

Un buon esempio della ricchezza delle informazioni di natura lessicale che si possono estrapolare dalle pagine del Glossario è dato dai modificatori della locuzione/sintagma nominale petia terre (e della variante grafica pecia terre), che per comodità abbiamo riassunto all'interno della Tabella 1:

Tabella 1. Modificatori del sintagma nominale petia terræ allinterno del Glossario

\begin{tabular}{|c|c|}
\hline $\begin{array}{l}\text { Aggettivo (alla forma f. sing.) } \\
\text { e occorrenza più antica nel } \\
\text { Glossario }\end{array}$ & (Eventuale) glossa o commento del Tiraboschi \\
\hline \multicolumn{2}{|l|}{$\begin{array}{l}\text { arativa (a. 1217) / aratoria (a. } \\
\text { 805) }\end{array}$} \\
\hline \multicolumn{2}{|l|}{ arva (a. 1202) } \\
\hline \multicolumn{2}{|l|}{ breniata (a. 1384) } \\
\hline \multicolumn{2}{|l|}{ bruchata / brugata (a. 1162) } \\
\hline \multicolumn{2}{|l|}{ buschiva / buscheliva (a. 1257) } \\
\hline \multicolumn{2}{|l|}{ campiva (a. 805) } \\
\hline \multicolumn{2}{|l|}{ caregiata (a. 1366) } \\
\hline \multicolumn{2}{|l|}{ casata (a. 1253) } \\
\hline castaneata (a. 1370) & Tiraboschi (1873: s.v. Terê): 'castagnata, piantata a castagni' \\
\hline \multicolumn{2}{|l|}{ castellata (a. 1245) } \\
\hline \multicolumn{2}{|l|}{ cerita (a. 1291) } \\
\hline \multicolumn{2}{|l|}{ clausoriba (a. 932) } \\
\hline \multicolumn{2}{|l|}{ copata (a. 1458) } \\
\hline corniva / cornata (a. 1257) & Tiraboschi (1873: s.v. Terê): 'che ha delle rocce' \\
\hline \multicolumn{2}{|l|}{ curizata (a. 1397) } \\
\hline dragonata (sec. XV) & $\begin{array}{l}\text { Dicesi del terreno franato o che facilmente frana; Franoso. } \\
\text { Nella V[alle] S[an] M[artino] è tuttora vivo dràc e nella Val } \\
\text { G[andino] Dragù nel signif[icato] di piana, scoscendimento }\end{array}$ \\
\hline \multicolumn{2}{|l|}{ fossadata (a. 1470) } \\
\hline guastiva (a. 1381) & Tiraboschi (1873: s.v. Terê): 'incolta' \\
\hline \multicolumn{2}{|l|}{ lobiata (a. 1439) } \\
\hline \multicolumn{2}{|l|}{ integra et inlibata (a. 879) } \\
\hline maroniva (a. 1473) & \\
\hline
\end{tabular}


(C) Italiano LinguaDue 2. 2021. F. Guerini, Appuntiper una edizione del Glossario Bergamasco Medioevale di Antonio Tiraboschi

\begin{tabular}{|c|c|}
\hline moina (a. 1368) & Aggettivo di terreno che vale 'molle, molliccio, acquoso' \\
\hline \multicolumn{2}{|l|}{ murata (a. 1223) } \\
\hline pasculiva (a. 1484) & Tiraboschi (1873: s.v. Terê): 'da pascolo' \\
\hline \multicolumn{2}{|l|}{ plodata (a. 1222) } \\
\hline prativa (a. 1107) & Tiraboschi (1873: s.v. Terê): 'ridotta a prato’' \\
\hline rivata (a. 1291) & A pendio (bergamasco riàt), con riva \\
\hline rosata (a. 1370) & 'Tiraboschi (1873: s.v. Rosada): 'rugiadosa, ricca di acqua' \\
\hline roboreta (a. 1104) & Tiraboschi (1873: s.v. Roér): 'sassosa, smossa, scoscesa' \\
\hline \multicolumn{2}{|l|}{ ronchiva (a. 1475) } \\
\hline scuriziva (a. 1287) & Tiraboschi (1873: s.v. Terê): 'soda, incolta, non lavorata' \\
\hline \multicolumn{2}{|l|}{ sedumata (sec. XIII) } \\
\hline \multicolumn{2}{|l|}{ solerata (a. 1209) } \\
\hline sortiva (a. 1497) & $\begin{array}{l}\text { Tiraboschi (1873: s.v. Medilif): 'Aggettivo di terreno che nella } \\
\text { valle Imagna e nella valle Brembana inferiore corrisponde a } \\
\text { Sortif di altri luoghi della provincia e vale: Roccioso con brevi } \\
\text { tratti dove cresce fieno magro' }\end{array}$ \\
\hline \multicolumn{2}{|l|}{ spondiva (a. 1453) } \\
\hline \multicolumn{2}{|l|}{ strameziva (a. 1484) } \\
\hline \multicolumn{2}{|l|}{ travata (a. 1458) } \\
\hline vangiva (a. 1475) & $\begin{array}{l}\text { Tiraboschi (1873: s.v. Vangbif): 'da vanga, che si lavora con la } \\
\text { vanga' }\end{array}$ \\
\hline vidata (a. 1244) & Tiraboschi (1873: s.v. Terê): 'coltivata a vigna' \\
\hline zerbiva (a. 1466) & Tiraboschi (1873: s.v. Terê): 'soda, incolta, sterile’ \\
\hline rovata (a. 1442) & Sinonimo di jugata: 'palancata, stecconata' \\
\hline
\end{tabular}

Alcuni aggettivi non sono esplicitamente glossati dal Tiraboschi, poiché il loro significato è trasparente e facilmente deducibile dal contesto nel quale si trovano inseriti: si pensi alle forme arva, buschiva / buscheliva, casata, castellata, fossadata, integra et inlibata, ronchiva, per citare solo alcuni esempi. In altri casi, l'assenza di glosse o commenti è riconducibile al carattere palesemente sommario, provvisorio, degli appunti di cui il Glossario si compone, che Tiraboschi non doveva certo considerare idonei alla pubblicazione nella forma in cui ci sono pervenuti. Come si può osservare nella Tabella 1 , la variante dialettale ottocentesca di molti degli aggettivi elencati è richiamata nel Vocabolario dei dialetti bergamaschi antichi e moderni alla voce teré, dove si elencano una quarantina di attributi del sostantivo terreno.

Per quanto concerne infine le annotazioni del terzo sottogruppo, ovvero, le osservazioni di carattere onomastico, occorre ricordare che presso la Biblioteca civica Angelo Mai sono conservate due opere manoscritte, la raccolta di nomi locali medioevali (MMB 8-22) e la raccolta di nomi personali medioevali (MMB 7), nelle quali Tiraboschi aveva verosimilmente pensato di far confluire buona parte dei materiali di carattere onomastico e toponomastico radunati nel corso delle sue ricerche. Le pagine del Glossario, tuttavia, contengono un certo numero di osservazioni e di spunti interessanti, spesso 
accompagnati da un rimando alle opere appena citate. Alla voce Lamivus (REW § 4862), ad esempio, leggiamo:

(11) Lamivus, - a Agg[ettivo] di terreno che vale Paludoso, Acquoso. Nella V[alle] $\mathrm{Br}$ [embana] (oltre la Goggia) dicesi tuttora Lama per prato ed è nome di parecchie nostre località. Vedi i miei Nomi locali.

Apprendiamo dunque che il micro-toponimo Lama contiene importanti informazioni sulla morfologia del luogo designato: in epoca medioevale il suo significato, attualmente del tutto opacizzato, doveva essere in larga misura trasparente e facilmente riconducibile all'aggettivo lamivus, attributo di terreno ${ }^{23}$. Esempi simili a quello appena citato testimoniano come, alla base di numerosi toponimi e microtoponimi di tradizione orale, vi siano elementi naturali del paesaggio ritenuti salienti dagli appartenenti alla comunità locale e per questo prescelti come punti di orientamento nella mappatura del territorio: una siepe di frassino (12a), un terreno sabbioso (12b), una vigna (12c), diventano così componenti di un sapere condiviso, attraverso il quale una comunità si appropria del territorio stesso:
a. "in territ[orio] Stazani ad cesam de frasceno" (MMB 24, s.v. Cesa)
b. "in terrat[orio] de Mapello [...] ubi dicitur prope geram" (MMB 24, s.v. Cerbivus)
c. "in territ[orio] de Somascha [...] ubi dicitur ad vitem de la ripa vegia"
(MMB 26, s.v. Vegia)

Altri micro-toponimi fanno invece riferimento ad interventi di antropizzazione del territorio, che assumono con il passare del tempo una funzione identificativo-referenziale: così, in una carta dell'anno 1194, leggiamo «[...] prope portam monasterii de Lavate ubi dicitur in platea de la zusterna» (MMB 26, s.v. Zusterna); altrove l'elemento più saliente per la localizzazione di una certa porzione del territorio può essere rappresentato da una cava ("[...] ibi ubi dicitur subtu cava de Alzano", MMB 25, s.v. Pestonus») oppure da una fontana («in Sedrina ubi dicitur ad fontanellam de l'albio», MMB 24, s.v. Fontanella).

Per quanto riguarda invece i nomi di persona, nei frammenti citati all'interno del Glossario abbondano gli antroponimi di origine germanica (Arnulfus, Launecbild, Amaldus, Gundepert, per mezionare solo alcuni esempi), a dimostrazione, se ce ne fosse bisogno, di come due secoli di presenza longobarda in Lombardia abbiano lasciato profonde tracce nel patrimonio onomastico della popolazione locale. Frequenti sono pure, prevedibilmente, gli antroponimi di tradizione cristiana, ispirati a santi venerati dalla popolazione (Georgius, Iohannes, Petrus, Paulus ...) o più genericamente beneauguranti (Venturinus, Benedictus, Benignus, ...), nonché i nomi laici di tradizione latina (Leoninus, Marinus, Costantius, Gratianus, ...). Ma le forme di maggiore interesse, che non passano inosservate allo sguardo attento del Tiraboschi linguista, sono i numerosi soprannomi, spesso inseriti in formule del tipo 'qui $X$ vocitatur' o 'qui $X$ dicitur', di cui di seguito portiamo alcuni esempi:

$$
\begin{aligned}
& \text { D[ominus] Bonaventura Bocadesurice (MMB 23, s.v. Boca) } \\
& \text { Guarischi de Stabullo Crescinbene (MMB 23, s.v. Bagothus) } \\
& \text { Domenginus dictus Marcharius (MMB 24, s.v. Draperius) } \\
& \text { Joh[annes] qui dicitur Formiga (MMB 24, s.v. Formiga) } \\
& \text { Lanfrancus Gratamascherpe (MMB 25, s.v. Mascherpa) } \\
& \text { Lanfranci Pesamigole (MMB 26, s.v. Taverna) }
\end{aligned}
$$

${ }^{23} \mathrm{Si}$ noti, en passant, che l'aggettivo lamivus non è incluso nella Tabella 1 alla pagina precedente, poiché all'interno del Glossario non appare utilizzato per modificare la locuzione petia terra. 
Tali soprannomi (che in origine dovevano essere semanticamente trasparenti e alludere a caratteristiche fisiche e/o tratti psicologici e caratteriali del portatore), testimoniano la graduale entrata in crisi del sistema onomastico a nomen unicum diffusosi in territorio italiano a cominciare dalla fine del IV secolo, che De Felice (1982: 134) descrive come «l'anello di congiunzione, la zona di saldatura tra l'ultimo sistema onomastico latino e il nuovo sistema italiano del nome individuale, che resta nome unico per tutto l'alto medioevo». Proprio nel corso dell'alto medioevo, il progressivo logoramento della capacità distintiva dei nomi unici fa sì che questi ultimi inizino ad essere accostati a forme che, in un primo momento, hanno la sola funzione di facilitare l'identificazione del portatore, ma col tempo sono destinate a diventare ereditarie, prefigurando il definitivo fissarsi del cognome, che avrà luogo a partire dall'inizio dell'età moderna. Tra queste forme, accanto a patronimici (ad es., Zocchinus filius Guilelmi, Belfanti de Bertoldis, ...) e etnici (Paulus de Vaprio, Ayulfus de Brembate, ...), si distinguono per originalità e varietà di composizione dei materiali lessicali proprio i soprannomi. Si tratta di un argomento al quale qui possiamo solo accennare ${ }^{24}$, ma che fornisce un'ulteriore testimonianza dei molteplici spunti di riflessione e di ricerca che le pagine del Glossario possono offrire al lettore.

\section{CONCLUSIONI}

A conclusione di questa prima, sommaria disamina dei preziosi materiali di cui il Glossario si compone, ci auguriamo di essere riusciti ad illustrare come la realizzazione di una edizione (cartacea e/o digitale) di tali appunti possa mettere a disposizione della comunità scientifica - linguisti, dialettologi, filologi, ma anche studiosi della cultura giuridica, legislativa e amministrativa medioevale - un data base di materiali accuratamente controllati sul versante linguistico-filologico e fruibili per molteplici opzioni di ricerca. Si tratta di documenti molto eterogenei (testi statutari, atti notarili, lasciti e donazioni), che Tiraboschi giudicava accomunati dalla possibilità di gettare luce sulle fasi più antiche della storia del dialetto bergamasco, testimoniandone lo sviluppo a partire dalle forme latine, secondo le direttrici fondamentali di ricerca della dialettologia romanza, che proprio nella seconda metà dell'Ottocento forniva alla comunità scientifica alcuni dei suoi contributi più importanti.

Scriveva Adolfo Mussafia al collega ed amico Graziadio Isaia Ascoli in una lettera datata 8 luglio 1886: «[...] voi mi avevate fatto cenno di un altro glossario [latino bergamasco] posseduto da Tiraboschi, e dell'intenzione che questi aveva di pubblicarlo. Non ho mai inteso che egli abbia mandato ad effetto il suo divisamento; e ora leggo nel Giornale [Storico della Letteratura Italiana] che il valentuomo è morto. Ci sarebbe modo di sapere ove sia andato a finire il codice da lui posseduto e se ancora in mano dei suoi eredi, ci sarebbe la possibilità di acquistarlo?» ${ }^{25}$. Se anche il nostro giudizio ci ingannasse, crediamo che le parole di Mussafia possano convincentemente dimostrare come, tra $\mathrm{i}$ manoscritti inediti del Tiraboschi che ancora giacciono presso la Biblioteca civica Angelo Mai, il Glossario meriti di essere dato quanto prima alle stampe.

\footnotetext{
24 Per approfondimenti, si rimanda all'ampia trattazione di Emidio De Felice (1982: 132-154) e di Carla Marcato (2009: 89-104).

${ }^{25}$ Lettera pubblicata da Clemente Merlo in appendice ad un articolo dal titolo "L'Ascoli e i canoni della glottologia" (cfr. Merlo, 1931: 30).
} 
(C) Italiano LinguaDue 2. 2021. F. Guerini, Appuntiper una edizione del Glossario Bergamasco Medioevale di Antonio Tiraboschi

\section{RIFERIMENTI BIBLIOGRAFICI}

a) Opere citate da Antonio Tiraboschi nella versione manoscritta del Glossario Bergamasco Medioevale

A.A. V.V. (1873), Historice Patrice Monumenta. Tomus XIII. Codex Diplomaticus Langobardic, Augustæ Taurinorum, e Regio Typographeo.

Angelini G. B. (1724), Descrizione dell'uccellare col roccolo. Composta, e dedicata al genio de' Bergomaschi, ed alla curiosità de' Forestieri, nella Stamperia di Giovanni Santini, con facoltà de SUPERIORI, Bergamo.

Ascoli G. I. (1873), "Saggi ladini”, in Archivio Glottologico Italiano, I, pp. 1- 537.

Barsecchini D. (1841), Memorie e Documenti per servire allistoria del ducato di Lucca, Tomo V, Parte II, F. Bertini, Tipografo ducale, Lucca.

Casati C. (1872), Treviglio di Ghiara d'Adda e suo territorio. Memorie storico-statistiche, coi tipi della Perseveranza, Milano.

Celestino da Bergamo (1617), Historia Quadripartita di Bergomo e suo territorio. Parte Prima da diuersi auttori stampati, e manuscritti. Oue tronasi compreso quel tutto quasi, che vi è aunenuto, da che fù Bergomo edificato, fin à tempi nostri, \& molte cose notande, per Valerio Ventura, Bergamo.

D’Azeglio M. (1871), Scritti postumi. La lega lombarda. Scritti politici. Scritti vari. Epistolario, a cura di Ricci M., G. Barbèra Editore, Firenze.

Diez F. Ch. (1853), Etymologisches Wörterbuch der Romanischen Sprachen, Fünfte Ausgabe, Adolph Marcus, Bonn.

Du Cange Ch. (1883-87), Glossarium mediae et infimae latinitatis, L. Favre, Niort.

Fanfani P. (1870), Voci e maniere del parlar fiorentino, Tipografia del Vocabolario, Firenze.

Grassi G. (s.d.), Alcune notizie sulla V alle di Scalve scritte nel 1843, con aggiunte trascritte nel 1854, Manoscritto non pubblicato, conservato presso la Biblioteca Civica Angelo Mai di Bergamo.

Lampertico F. (1875), Il Lavoro, Fratelli Treves Editori, Milano.

Littré É. (1873), Dictionnaire de la langue française contenant: la nomenclature, la grammaire, la signification des mots, la partie historique, l'étymologie, Plusieurs Tomes, Librairie Hachette, Parigi.

Liverani F. (1875), Il ducato e le antichità longobarde e saliche di Chiusi, A. Mucci, Siena.

Luchaire, A. (1877), Les origines linguistiques de l'Aquitaine, Imprimerie et Litographie Veronese, Pau.

Lupo M. (s.d.), Excerpta ex Actis Notariorum Bergomi, Manoscritto non pubblicato, conservato presso la Biblioteca Civica Angelo Mai di Bergamo.

Lupo M. (1784), Codex diplomaticus civitatis, et ecclesice Bergomatis, Vol. I, ex Typographa Vincentii Antoine, Bergamo.

Lupo M. (1799), Codex diplomaticus civitatis, et ecclesice Bergomatis. Vol. II. Opus posthumum editum, ac monumentis, et commentariis actum a presbytero Josepho Ronchetti, ex Typographa Vincentii Antoine, Bergamo.

Mazzi A. (1877), Il Sextarius Pergami. Saggio di ricerche metrologiche, Tipografia Pagnoncelli, Bergamo.

Muratori L. A. (1751), Dissertarioni sopra le antichità italiane, già composte e pubblicate in latino dal proposto Lodovico Antonio Muratori, e da esso poscia compendiate e trasportate nell'italiana favella, 3 Voll. Opera postuma data in luce dal proposto Gian-Francesco Soli Muratori suo nipote. Milano, a spese di Giambatista Pasquali.

Peruzzi S. L. (1868), Storia del commercio e dei banchieri di Firenze in tutto il mondo conosciuto dal 1200 al 1345, Coi tipi di M. Cellini e C, Firenze.

Romanin S. (1853), Storia documentata di Venezia, Tomo I, Pietro Naratovich, Venezia.

Romanin S. (1854), Storia documentata di Venezia, Tomo II, Pietro Naratovich, Venezia. 
(C) Italiano LinguaDue 2. 2021. F. Guerini, Appuntiper una edizione del Glossario Bergamasco Medioevale di Antonio Tiraboschi

Rosa G. (1863), Statuti inediti della provincia di Bergamo anteriori al secolo XVI, Pagnoncelli, Bergamo.

Rota G. B. (1880), Il comune di Chiari e le sue memorie storiche, Tipografia di Giovanni Bersi, Brescia.

Sacchetti F. (1874), Le novelle recate a buona lezione e dichiarate con note, Edoardo Sonzogno Editore, Milano.

Valentini A. (1878), Liber poteris della città e del comune di Brescia, e la serie dei suoi console e podestà, Tipografia Francesco Apollonio, Brescia.

b) Opere consultate per la redazione del presente contributo

Belotti B. (1956), "Antonio Tiraboschi”, in Belotti B. Gli eccellenti bergamaschi (vol. I), Edizioni Orobiche, Bergamo, pp. 189-199.

Bondioli Magnati G. (1984), "Antonio Tiraboschi: cronologia della vita", in Bondioli Magnati G., Boninelli M. (a cura di), Opere a stampa di Antonio Tiraboschi. Con cronologia della vita e bibliografia critica, Quaderni dell'Archivio della Cultura di Base 5, Bergamo, Sistema Bibliotecario Urbano, Bergamo, pp. 11-33.

Bondioli Magnati G., Boninelli M. (a cura di) (1984), Opere a stampa di Antonio Tiraboschi. Con cronologia della vita e bibliografia critica, Quaderni dell'Archivio della Cultura di Base 5, Sistema Bibliotecario Urbano, Bergamo.

Boninelli M. (1985), "Antonio Tiraboschi: il «manovale di cultura» e la sua «officina»", in Vitali G., Bravi O. (a cura di), Lingue e culture locali. Le ricerche di Antonio Tiraboschi, Lubrina, Bergamo, pp. 52-68.

Brolis M. T., Brembilla G., Corato M. (a cura di) (2001), La matricola femminile della Misericordia di Bergamo, 1265-1339, École française de Rome, Roma.

Cortesi M. (1983), Statuti rurali e statuti di valle. La provincia di Bergamo nei secoli XIII-XVIII, (Fonti per lo studio del territorio bergamasco, III), Assessorato alla Cultura della Provincia di Bergamo, Bergamo.

De Felice E. (1982), I nomi degli italiani, Marsilio Editore, Venezia.

DELI = Dizionario Etimologico della Lingua Italiana, a cura di Manlio Cortelazzo e Paolo Zolli, Zanichelli, Bologna, 1979-1988.

DELL = Dictionnaire Étimologique de la Langue Latin. Histoire des mots, Alfred Ernout, Antoine Meillet, Quatrième édition revue corrigée et augmentée d'un index, Klincksieck, Paris, 1960.

Finazzi G. (1876), "Edizione di Antiquae collationes statuti veteris civitatis Pergami”, in Historiae Patriae Monumenta 16, Leges Municipales II, Torino, 1921-2046.

Forgiarini G. (1996), Lo statuto di Bergamo del 1353, Fonti Storico-giuridiche, Statuti II, CISAM, Spoleto.

Francovich Onesti, N. (2013), Vestigia longobarde in Italia (568-774). Lessico e antroponimia (seconda edizione aggiornata), Artemide, Roma.

Jarnut, J. (1980), Bergamo 568 - 1098. Storia istituæionale, sociale ed economica di una città lombarda nell'alto medioevo, Archivio Bergamasco, Centro Studi e Ricerche, Bergamo.

Leydi R. (a cura di) (1974), "Canti popolari bergamaschi. La raccolta di Antonio Tiraboschi. Con prefazione di Bonaventura Foppolo", in Cultura tradizionale in Lombardia 1. Bergamo e il suo territorio [Quaderni di documentazione regionale], Bergamo, pp. 201-285.

Little L. K. (1988), Libertà Carità Fraternità. Confraternite laiche a Bergamo nell'età del comune, Edizione degli statuti a cura di Sandro Buzzetti. Ricerca codicologica di Giulio Orazio Bravi, Lubrina, Bergamo.

Mantovani G. (1892), "Biografia sinottica del Prof. Antonio Tiraboschi compilata su documenti ufficiali", in Il Sentierone, numero unico, agosto-settembre, 11. 
(C) Italiano LinguaDue 2. 2021. F. Guerini, Appuntiper una edižone del Glossario Bergamasco Medioevale di Antonio Tiraboschi

Mazzi A. (1884), "Cenni biografici di illustri concittadini. Antonio Tiraboschi”, in Bergamo o sia Notizie Patrie. Almanacco scientifico-artistico-letterario per l'anno bisestile 1884, Pagnoncelli, Bergamo, pp. 51-73.

Marcato C. (2009), Nomi di persona, nomi di luogo. Introdurione all'onomastica italiana, il Mulino, Bologna.

Merlo C. (1931), "L’Ascoli e i canoni della glottologia. Con una postilla", in L'Italia Dialettale, VII, 1, pp. 1-32.

Padoa Schioppa A. (1984), "Brevi note sugli statuti bergamaschi e lombardi", in Cortesi M. (a cura di), Statuti rurali e statuti di valle. La provincia di Bergamo nei secoli XIII XVIII. Atti del Convegno. (Fonti per lo studio del territorio bergamasco, V), Provincia di Bergamo, Bergamo.

Passerini Tosi C. (1985), "Tiraboschi lessicografo. Per un vocabolario dei dialetti bergamaschi", in Vitali G., Bravi O. (a cura di), Lingue e culture locali. Le ricerche di Antonio Tiraboschi, Lubrina, Bergamo, pp. 183-201.

Pitré G. (1883), "Commemorazione”, in Archivio per lo Studio delle Tradizioni Popolari, vol. II, Luigi Pedone Lauriel Editore, Palermo, p. 476.

Prina B. (1883), "Commemorazione. Antonio Tiraboschi", in Arcbivio Storico Lombardo. Giornale della società Storica Lombarda, X, pp. 788-792.

Poletti R. M. (1942), "Antonio Tiraboschi folclorista e linguista bergamasco", in Bergomum XXXVI, fascicolo 1, pp. 1-18; fascicolo 2, pp. 21-40; fascicolo 3, pp. 53-78.

Putzu I. (2000), Il soprannome. Per uno studio multidisciplinare della nominazione, CUEC Editrice, Cagliari.

REW = Romanisches etymologisches Wörterbuch, herausgegeben von Wilhelm Meyer-Lübke, Carl Winter, Heidelberg, 1911.

Robecchi M. (2013), "Un inedito glossario Latino-Bergamasco del Trecento (ms. MAB 29)", in L'Italia Dialettale LXXIV (Serie Terza, X), pp. 85-133.

Rosa G. (1863), Statuti inediti della provincia di Bergamo anteriori al secolo XVI, Pagnoncelli, Bergamo.

Rosa G. (1869), Statuti di Vertova del 1235, del 1248, del 1256, con annotazioni, Tipografia Fratelli Fiori, Brescia.

Sanga G. (1987), "Bergamo tra Venezia e Milano", in Sanga G. (a cura di), Lingua e dialetti di Bergamo e delle valli. Tomo I: Il dialetto di Bergamo, Lubrina, Bergamo, pp. 17-35.

Scharf G. P. (2004), "Gli statuti duecenteschi di Vertova e Leffe", in Chiappa Mauri L. (a cura di), Statuti rurali lombardi del secolo XIII, Unicopli, Milano, pp. 91-104.

Simone R. (1997), "Esistono verbi sintagmatici in italiano?", in De Mauro T., Lo Cascio V. (a cura di), Lessico e grammatica. Teorie linguistiche e applicazioni lessicografiche, Bulzoni, Roma, pp. 155-170.

Statutorum Bergomi (1551), Repertorium copiosissimum statutorum Bergomi, Comin da Trino, Venezia.

Storti Storchi C. (1984), "Statuti viscontei di Bergamo", in Cortesi M. (a cura di), Statuti rurali e statuti di valle. La provincia di Bergamo nei secoli XII - XVII (Fonti per lo studio del territorio bergamasco, V), Assessorato alla Cultura della Provincia di Bergamo, Bergamo, pp. 51-92.

Storti Storchi C. (1986), Lo statuto di Bergamo del 1331, Fonti Storico-giuridiche, Statuti I, Giuffrè, Milano.

Storti Storchi C. (2007), Scritti sugli statuti lombardi, Giuffrè, Milano.

Tiraboschi A. (1859), Saggio di un vocabolario bergamasco, Tipografia Crescini, Bergamo.

Tiraboschi A. (1873), Vocabolario dei dialetti bergamaschi antichi e moderni, Tipografia dei fratelli Bolis, Bergamo [Riproduzione anastatica dell'originale, con le Appendici Prima e Seconda, Forni, Bologna 1980]. 
(C) Italiano LinguaDue 2. 2021. F. Guerini, Appuntiper una edizione del Glossario Bergamasco Medioevale di Antonio Tiraboschi

Tiraboschi A. (1880), "Cenni intorno alla Valle Gandino ed ai suoi Statuti (parte prima)", in Archivio Storico Lombardo. Giomale della società Storica Lombarda, VII, pp. 5-40.

Tiraboschi A. (1882), "Cenni intorno alla Valle Gandino ed ai suoi Statuti (parte seconda). A cui segue come saggio il Frammento dell'antico Statuto di Leffe", in Archivio Storico Lombardo. Giornale della società Storica Lombarda, IX, pp. 369-402.

Volpi V. (1977), "Nota Biografica a Fiabe Bergamasche. La raccolta inedita di Antonio Tiraboschi", in Leydi R. (a cura di), Bergamo e il suo territorio [Mondo popolare in Lombardia 1], Silvana, Milano, pp. 343-353.

Zonca A. (2010), "Le pergamene di Sancta Grata inter vites", in Quaderni di Archivio Bergamasco 4, pp. 13-38. 